\title{
COMPETITION MODEL IN A CHEMOSTAT WITH MONOTONE FUNCTIONAL RESPONSE
}

\author{
S. M. Sohel Rana \\ Department of Mathematics \\ University of Dhaka, Dhaka-1000, Bangladesh \\ Email: srana_math@univdhaka.edu
}

Received 21.04.09 Accepted 05.03.11

\begin{abstract}
In this paper, a model for competition of two populations of microorganisms in a chemostat with monotone functional response is considered. We prove that the solutions are positive and bounded for all time. Stability of nonnegative equilibria and persistence of solutions are presented. Graphical results are also given to help illustrate the key points in the population dynamics of the model.
\end{abstract}

Key words: competition; local stability; global stability; persistence.

\section{Introduction}

In mathematical biology, the chemostat is the best laboratory apparatus that can be used to study competition between different populations of microorganisms for a growth limiting nutrient and has the advantage that certain of the biological parameters presumed to influence competitive outcome can be controlled by the experimenter. In this paper, we consider the general form of the model done by Kapur [5] describing two populations of microorganisms competing for a single growth limiting nutrient in a chemostat. Rigorous mathematical analysis of the chemostat model for an arbitrary number of competitors with Michaelis-Menten type functional response has been carried out by Hsu [3] and Hsu, Hubbell, and Waltman [4]. A model of competition with discrete delay has been carried out by Wolkowicz and Xia [7]. The mathematical results in all of these papers indicate that competitive exclusion holds. A 'competitive exclusion principle', stating that in the long run only one population survives while the others die out. We assume that there is no direct interaction in each of the species. Our model consists of a set of three non-linear differential equations and predicts that at most one population survives and that the population that survives can be predicted based on the relative values of the break-even concentrations, parameters that can be determined by growing each population alone in the chemostat and measuring the steady state concentration of the nutrient.

\section{Model equations}

We shall consider the following model of two populations of microorganisms competing exploitatively for a single, essential, nonreproducing growth limiting nutrient in a well- 
stirred chemostat:

$$
\begin{aligned}
& s^{\prime}(t)=D\left(s^{0}-s(t)\right)-\sum_{i=1}^{2} \frac{1}{\gamma_{i}} f_{i}(s(t)) p_{i}(t) \\
& p_{i}^{\prime}(t)=\left(-D+f_{i}(s(t))\right) p_{i}(t) \\
& s(0)=s_{0}>0, p_{i}(0)=p_{i 0}>0, i=1,2 .
\end{aligned}
$$

In system (1), $s(t)$ denotes the concentration of nutrient and $p_{i}(t)$ denotes the concentration of the $i$ th population of microorganism in the chemostat at time $t ; f_{i}(s) / \gamma_{i}$ represents the nutrient uptake function for the $i$ th population; we assume that $f_{i}(s)$ represents the per-capita growth rate of the $i$ th population as a function of nutrient concentration and so $\gamma_{i}$ is a growth yield constant; $s^{0}$ and $D$ are positive constants and denote respectively the concentration of the growth limiting nutrient in the fresh inflowing medium and the flow rate of the chemostat. It is assumed that the individual death rate of any species is insignificant, compared to the flow rate, and hence can be ignored.

We make the assumptions below on the response functions $f_{i},(\mathrm{i}=1,2)$ in model $(1)$ :

$f_{i}: R_{+} \rightarrow R_{+}$is continuously differentiable and $f_{i}(0)=0$;

$f_{i}$ is monotonically increasing;

there exists a unique (possibly extended) real number $0<\lambda_{i}<\infty$ such that

$$
\begin{aligned}
& f_{i}(s)<D \quad \text { if } s<\lambda_{i} \\
& f_{i}(s)>D \text { if } s>\lambda_{i} \\
& \lim _{s \rightarrow \infty} f_{i}(s)<\infty
\end{aligned}
$$

Here, the value $\lambda_{i}$ represents the break-even concentration of the nutrient for the $i$ th population and it has played an important role in determining competitive ability.

Now it is convenient to pass to non-dimensional variables $\bar{s}:=s / s^{0}$ and $\bar{p}_{i}:=p_{i} /\left(\gamma_{i} s^{0}\right)$. After dropping the bars and writing $f_{i}(s)$ instead of $f_{i}\left(\bar{s}^{0}\right)$, we obtain

$$
\begin{aligned}
& s^{\prime}(t)=D(1-s(t))-\sum_{i=1}^{2} f_{i}(s(t)) p_{i}(t) \\
& p_{i}^{\prime}(t)=\left(-D+f_{i}(s(t))\right) p_{i}(t) \\
& s(0)=s_{0}>0, p_{i}(0)=p_{i 0}>0, i=1,2 .
\end{aligned}
$$


It is observed that the 'new' $f_{i}(s)$ also satisfy the properties (2).

Note that in the definition of (1), we assume $p_{i 0}>0$ for $i=1,2$. This involves no loss of generality since if $p_{i 0}=0$ for $i=1,2$, then $p_{i}(t)=0$ for all $t \geq 0$ and that population can be eliminated from consideration.

\section{Preliminary results}

We first observe that the right hand side of the system (3) is a smooth function of the variable $\left(s, p_{1}, p_{2}\right)$. So local existence and uniqueness properties hold in the region $\left\{\left(s, p_{1}, p_{2}\right) \in R_{+}^{3} \mid p_{i}>0, i=1,2\right\}$. Now we shall prove that (3) has positive and bounded solutions, which is a prerequisite for any model of the chemostat.

Lemma 3.1: Let $a \in(-\infty, \infty)$ and $f:[a, \infty] \rightarrow R$ be a differentiable function. If $\lim _{t \rightarrow \infty} f(t)$ exists (finite) and the derivative function $f^{\prime}(t)$ is uniformly continuous on $(a, \infty)$, then $\lim _{t \rightarrow \infty} f^{\prime}(t)=0$.

The proof of this lemma due to Barbalat can be found in Gopalsamy [2].

Lemma 3.2: All the solutions $s(t), p_{i}(t),(i=1,2)$ of system (3) are positive and bounded for $t>0$. Moreover, if $\lambda_{i}<1,(i=1,2)$, then $s(t)<1$ for all sufficiently large $t$.

Proof: Suppose it is not true that $s(t)>0$ for all $t>0$. Let $t_{1}=\min \{t: t>0 \& s(t)=0\}$. Then $s(t)>0, \forall t \in\left[0, t_{1}\right)$. But from the first equation of (3), we have $s^{\prime}\left(t_{1}\right)=D>0$. That is, $s^{\prime}(t)>0$ on a neighborhood of $t_{1}$. This implies there exists $\varepsilon>0$ such that $s(t)$ is increasing on $\left(t_{1}-\varepsilon, t_{1}+\varepsilon\right)$. Therefore, we have $0<s\left(t_{1}-\varepsilon / 2\right)<s\left(t_{1}\right)$, a contradiction. Thus $s(t)>0$ for all $t>0$. Again, from the second equation of (3), we have

$p_{i}^{\prime}(t)=\left(-D+f_{i}(s(t))\right) p_{i}(t), i=1,2$. This gives

$p_{i}(t)=p_{i 0} \exp \left[\int_{0}^{t}\left(-D+f_{i}(\mathrm{~s}(\theta))\right) \mathrm{d} \theta\right]$, which shows that $p_{i}(t)>0$ for all $t \geq 0$.

Therefore, the system (3) with positive initial conditions at $t=0$ produces positive solution for $t>0$.

We set 


$$
v(t)=1-s(t)-\sum_{i=1}^{2} p_{i}(t) \text { for all } t \geq 0,
$$

where $s(t), p_{i}(t), i=1,2$ are arbitrary positive solutions of (3). Then it follows from (3) that

$$
v^{\prime}(t)=-D v(t) \text { for all } t \geq 0
$$

and consequently,

$$
s(t)+\sum_{i=1}^{2} p_{i}(t)=1+\varepsilon(t), t \geq 0,
$$

where $\varepsilon(t) \rightarrow 0$ exponentially as $t \rightarrow \infty$.

Since all solutions are positive for all $t>0$, it follows from (4) that all solutions are bounded.

We now prove that under the condition $\lambda_{i}<1, s(t)<1$ for all sufficiently large $t$. First note that if $s(\bar{t})=1$ for some $\bar{t} \geq 0$, then $s^{\prime}(\bar{t})<0$ and so if $s(T) \leq 1$ for some $T \geq 0$ then $s(t)<1$ for all $t>T$.

Suppose that $s(t)>1$ for all large $t$. Then $s^{\prime}(t) \leq(1-s(t)) D<0$ and so $s(t) \downarrow s^{*} \geq 1>\lambda_{i}$ for some $s^{*}$ as $t \rightarrow \infty$. Since $s(t)$ is bounded below and satisfies (3), we must have $s^{*}=1$. Thus $s(t)>\lambda_{i}$ for all large $t$. Then (3) and (2) imply that

$$
p_{i}^{\prime}(t)=\left(-D+f_{i}(s(t))\right) p_{i}(t)>0
$$

for all large $t$. Therefore $p_{i}(t) \uparrow p_{i}^{*}>0$ exists as $t \rightarrow \infty$. From (5), we see that $p_{i}^{\prime}(t)$ is uniformly continuous on $[0, \infty)$, since $s(t), p_{i}(t), s^{\prime}(t)$ and $p_{i}^{\prime}(t)$ are all bounded for all $t>0$. Hence by Lemma 3.1, $\lim _{t \rightarrow \infty} p_{i}^{\prime}(t)=0$. Since $\lim _{t \rightarrow \infty} s(t)=1>\lambda_{i}$, it follows that $\lim _{t \rightarrow \infty} p_{i}(t)=0$, by (5). But this contradicts $p_{i}(t) \uparrow p_{i}^{*}>0$ as $t \rightarrow \infty$. This completes the proof.

Theorem 3.3: For every positive solution of (3), if $\lambda_{i} \geq 1, \quad(i=1,2)$, then $\lim _{t \rightarrow \infty} p_{i}(t)=0$.

Proof: By an argument similar to the one given in Lemma 3.2, it follows that either $s(t) \downarrow 1$ as $t \rightarrow \infty$ or $s(t)<1$ for all sufficiently large $t$. Suppose that $s(t) \downarrow 1$ as $t \rightarrow \infty$. Since $s(t), p_{i}(t)$ and their derivatives $s^{\prime}(t), p_{i}^{\prime}(t)$ are bounded, from (3), $s^{\prime}(t)$ is uniformly continuous. By Lemma 3.1, $\lim _{t \rightarrow \infty} s^{\prime}(t)=0$ and consequently, 


$$
\lim _{t \rightarrow \infty} \sum_{j=1}^{2} p_{j}(t) f_{j}(s(t))=0
$$

This leads to $\lim _{t \rightarrow \infty} p_{i}(t)=0$, as desired.

Suppose now that $s(t)<1 \leq \lambda_{i}$ for all large $t$. Then (2) gives

$$
p_{i}^{\prime}(t)=\left(-D+f_{i}(s(t))\right) p_{i}(t) \leq 0
$$

for all large $t$ and so $p_{i}(t) \downarrow p_{i}^{*} \geq 0$ as $t \rightarrow \infty$. As in Lemma 3.2, it can be shown that $\lim _{t \rightarrow \infty} p_{i}^{\prime}(t)=0$ and by $(6)$, it then follows that

$$
\lim _{t \rightarrow \infty}\left(-D+f_{i}(s(t))\right) p_{i}(t)=0
$$

If there is a sequence $\left\{t_{m}\right\} \uparrow \infty$ such that $\lim _{m \rightarrow \infty} p_{i}\left(t_{m}\right)>0$, then (7) forces $\lim _{m \rightarrow \infty} f_{i}\left(s\left(t_{m}\right)\right)=D$, leading to $\lim _{m \rightarrow \infty} s\left(t_{m}\right)=\lambda_{i}$. But $s\left(t_{m}\right)<1$ for large $m$ and $\lambda_{i} \geq 1$. This is a contradiction if $\lambda_{i}>1$. When $\lambda_{i}=1$, a contradiction can also be derived by using (4) and the fact that $\lim _{m \rightarrow \infty} s\left(t_{m}\right)=\lambda_{i}=1$. This shows that $\lim _{t \rightarrow \infty} p_{i}(t)=0$ and this completes the proof.

\section{Equilibria}

System (3) has the following equilibria

$E_{0}(1,0,0), E_{1}\left(\lambda_{1}, 1-\lambda_{1}, 0\right), E_{2}\left(\lambda_{2}, 0,1-\lambda_{2}\right)$

where the break-even concentration $\lambda_{i},(i=1,2)$ is defined as the unique solution of

$$
f_{i}(s)-D=0 \text { (if it exists) }
$$

and an interior equilibrium $\hat{E}\left(\hat{s}, \hat{p}_{1}, \hat{p}_{2}\right)$

where $\hat{s}$ is defined as the unique solution of $f_{1}(s)=f_{2}(s)=D$ and $\hat{p}_{1} \& \hat{p}_{2}$ satisfy the equation $p_{1}+p_{2}=1-\hat{s}$ with $\hat{s} \in(0,1)$.

The washout equilibrium point $E_{0}$ always exists.

The necessary and sufficient condition for the existence of $E_{i}$ is $\lambda_{i}<1, i=1,2$.

Finally, it can be easily shown that $\hat{E}$ exists and is unique if $\hat{p}_{i}>0, i=1,2$. 


\section{Stability analysis}

Here we shall investigate the local stability of the equilibrium points $E_{0}, E_{1}, E_{2}$ and $\hat{E}$ by finding the eigenvalues of the associated Jacobian matrices.

System (3) is persistent, i.e., all populations coexist at a nonzero level if all the boundary equilibria are repellers.

The Jacobian matrix of (3) takes the form

$$
J\left(s, p_{1}, p_{2}\right)=\left(\begin{array}{ccc}
-D-f_{1}^{\prime}(s) p_{1}-f_{2}^{\prime}(s) p_{2} & -f_{1}(s) & -f_{2}(s) \\
f_{1}^{\prime}(s) p_{1} & f_{1}(s)-D & 0 \\
f_{2}^{\prime}(s) p_{2} & 0 & f_{2}(s)-D
\end{array}\right)
$$

\subsection{Local stability and persistence}

Now we are positive to prove the following result.

\section{Theorem 5.1:}

If $\lambda_{i}>1, i=1,2$

Then $E_{0}$ is locally asymptotically stable (LAS) and the system (3) is not persistent.

If $0<\lambda_{1}<\lambda_{2}<1$

Then $E_{1}$ is LAS in the $s-p_{1}$ plane and the system (3) is not persistent.

If $0<\lambda_{2}<\lambda_{1}<1$

Then $E_{2}$ is LAS in the $s-p_{1}$ plane and the system (3) is not persistent.

If $\hat{p}_{i}>0, i=1,2$

Then $\hat{E}$ is LAS in the positive octant.

Proof: For $E_{0}$, the eigenvalues of the Jacobian matrix (8) are $-D$, and $f_{i}(1)-D, i=1,2$. By Eq. (9), they are all negative. So $E_{0}$ is LAS and the system (3) is not persistent.

For $E_{1}$, the eigenvalues of the Jacobian (8) are

$-D<0, f_{2}\left(s_{1}\right)-D<0$ and $\left.-f_{1}^{\prime}\left(s_{1}\right)\left(1-s_{1}\right)\right)<0$ by Eq. (10)

Therefore $E_{1}$ is LAS in the $s-p_{1}$ plane and the system (3) is not persistent.

For $E_{2}$, the eigenvalues of (8) are 
$-D<0, f_{1}\left(s_{2}\right)-D<0$ and $\left.-f_{2}^{\prime}\left(s_{2}\right)\left(1-s_{2}\right)\right)<0$ by Eq. (11)

Therefore $E_{2}$ is LAS in the $s-p_{2}$ plane and the system (3) is not persistent.

For $\hat{E}$, the eigenvalues of the Jacobian (8) are

$0,-D<0$ and $-f_{1}^{\prime}(s) p_{1}-f_{2}^{\prime}(s) p_{2}<0$ by Eq. (12)

Thus $\hat{E}$ is LAS and the system (3) locally persistent. Since one of the eigenvalues is zero and other two are negative so if the position of interior equilibrium point $E_{3}$ is disturbed, it returns to another position of boundary equilibrium point.

\subsection{Global stability results}

In this section, we shall show that $E_{0}$ is globally asymptotically stable if only $E_{0}$ exists. The proof is very straightforward. If $E_{0}$ and $E_{1}$ (or $E_{2}$ ) exist, then $E_{1}$ (or $E_{2}$ ) is globally asymptotically stable. The proofs involve the construction of a Lyapunov function and the application of the Lyapunov-LaSalle theorem.

The following theorem shows the extinction of both populations, i.e., $E_{0}$ is a global attractor.

Theorem 5.2: If (9) holds, then all solutions of (3) satisfy $\lim _{t \rightarrow \infty}\left(s(t), p_{1}(t), p_{2}(t)\right)=(1,0,0)$.

Proof: It is a direct consequence of Theorem 3.3 and formula (4).

Our next theorem shows that competitive exclusion holds and only one competitor survives.

Theorem 5.3: If (10) holds, then all solutions of (3) satisfy

$\lim _{t \rightarrow \infty}\left(s(t), p_{1}(t), p_{2}(t)\right)=\left(\lambda_{1}, 1-\lambda_{1}, 0\right)$.

Proof: We choose a constant $\alpha>0$ satisfying $\max _{0<s<\lambda_{1}} w(s) \leq \alpha \leq \min _{\lambda_{2}<s<1} w(s)$

where $w(s)=\frac{f_{2}(s)\left(f_{1}(s)-D\right)\left(1-\lambda_{1}\right)}{D\left(f_{2}(s)-D\right)(1-s)}$

Define the Lyapunov function $V\left(s, p_{1}, p_{2}\right)$ as follows

$$
V=\int_{\lambda_{1}}^{s} \frac{\left(f_{1}(\xi)-D\right)\left(1-\lambda_{1}\right)}{D(1-\xi)} d \xi+\int_{p_{1}^{*}}^{p_{1}} \frac{\xi-p_{1}^{*}}{\xi} d \xi+\alpha p_{2}
$$


on the set $\Psi=\left\{\left(s, p_{1}, p_{2}\right): s \in(0,1), p_{i}>0, i=1,2\right\}$, where $p_{1}^{*}=1-\lambda_{1}$. Then the time derivative of $V$ along solutions of the differential equation is

$\dot{V}=p_{1}\left(f_{1}(s)-D\right)\left(1-\frac{\left(1-\lambda_{1}\right) f_{1}(s)}{D(1-s)}\right)+p_{2}\left(\alpha\left(f_{2}(s)-D\right)-\frac{f_{2}(s)\left(f_{1}(s)-D\right)\left(1-\lambda_{1}\right)}{D(1-s)}\right)$

First note that the first term in $\dot{V}$ is always nonpositive for $0<s<1$ and equals 0 for $s \in[0,1)$ if and only if $s=\lambda_{1}$ or $p_{1}=0$.

Define $h(s)=\alpha\left(f_{2}(s)-D\right)-\frac{f_{2}(s)\left(f_{1}(s)-D\right)\left(1-\lambda_{1}\right)}{D(1-s)}$.

If $s \in\left[\lambda_{1}, \lambda_{2}\right]$, then $h(s)<0$ for any choice of $\alpha>0$. Therefore, by definition of $\alpha$ satisfying (13), $h(s)<0$ for every $s \in(0,1)$. By Lemma 3.2 every bounded solution of (3) is contained in $\Psi$, and hence by LaSalle extension theorem [6] every solution of (3) approaches the set $M$, the largest invariant subset of $\Phi=\left\{\left(s, p_{1}, p_{2}\right) \in \bar{\Psi}: \dot{V}=0\right\} . \Phi$ is made up of points of the following forms

$$
\begin{aligned}
& (s, 0,0), \text { where } s \in[0,1] \\
& \left(\lambda_{1}, p_{1}, 0\right) \text {, where } p_{i} \in[0, \infty) .
\end{aligned}
$$

Since $V$ is bounded above, any point of the form $(s, 0,0)$ can not be in the $\omega$-limit set $\Omega$ of any solution initiating in the interior of $R_{+}^{3}$. The unique point of the form $\left(\lambda_{1}, p_{1}, 0\right) \in M$ implies that $s(t)=\lambda_{1}$ and $p_{2}(t)=0$, which in turn leads to $0=s^{\prime}(t)=D\left(1-\lambda_{1}\right)-f\left(\lambda_{1}\right) p_{1}$ and hence $p_{1}=1-\lambda_{1}$. Therefore $M=\left\{E_{1}\right\}$. This completes the proof.

Theorem 5.4: If (11) holds, then all solutions of (3) satisfy

$\lim _{t \rightarrow \infty}\left(s(t), p_{1}(t), p_{2}(t)\right)=\left(\lambda_{2}, 0,1-\lambda_{2}\right)$.

The proof follows by similar argument in Theorem 5.3.

\section{Discussion}

In this paper, we considered a model of exploitative competition for a single, essential, nonreproducing growth limiting nutrient. Kapur [5] carried out the equilibrium and extinction criteria for the competing populations in the case that the response functions are modeled by Michaelis-Menten dynamics. We assumed that the functional response is 
general monotone function. For this response function we proved that the outcome of competition for nutrient depends on the relative values of the break-even concentration. It is known (see Hsu [3] and Hsu, Hubbell, and Waltman [4]) that in chemostat models all species concentration eventually approach equilibrium concentration and at most one population avoids extinction, that is the competitive exclusion principle (see Armstrong and McGehee [1]) occurs. We found that if the break-even concentration $\lambda_{i}$ of the population $p_{i}$ is larger than one then that population dies out whether or not there is other competitor. If $E_{0}$ is the only equilibrium point, we showed that $E_{0}$ is a global attractor (this happens if $\lambda_{i}>1$ ). When $E_{0}, E_{1}$ or $E_{2}$ are the equilibrium points, we found that $E_{1}$ is LAS (for $0<\lambda_{1}<\lambda_{2}<1$ ). By constructing a Lyapunov function, we were able to show that $E_{1}$ is globally asymptotically stable, i.e., the population that survives is the one with the lowest break-even concentration. We also showed that if $\hat{E}$ exists then it is asymptotically stable.

Finally, for the Holling type III

$$
f_{i}(s)=\frac{m_{i} s^{2}}{\left(b_{i}+s\right)\left(c_{i}+s\right)}
$$

(multiple saturation) functional response (Fig-1), initial values, and parameters, numerical simulations show that solutions are converging to the positive equilibrium in the model (Fig-2). However, if the two populations only compete indirectly for the nutrient and do not compete directly each other, then only one population can survive (Fig-3, 4). This is similar to the phenomenon observed in chemostat models (see Hsu, Hubbell, and Waltman [4]). Both populations can also die out (Fig-5).

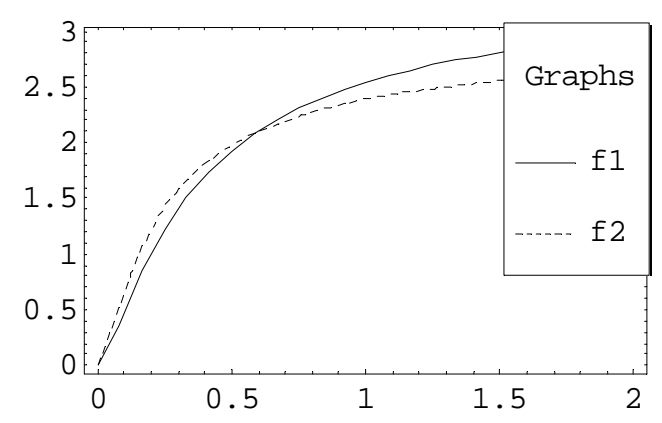

Fig-1: Graphical depiction of response functions $f_{i}$ with

$$
m_{1}=3.5, b_{1}=.20, c_{1}=.15, m_{2}=2.94, b_{2}=.12, c_{2}=.10 \text {. }
$$




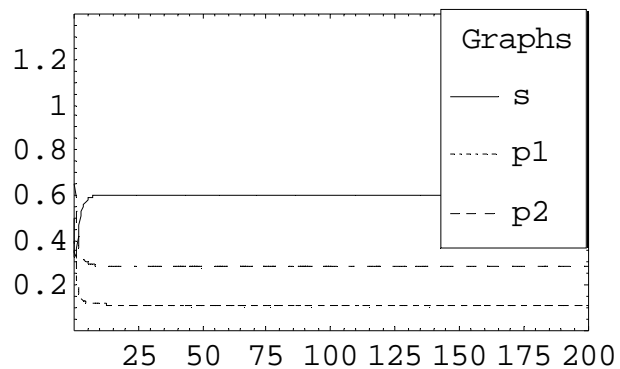

Fig-2: $D=2.1$, $\left(s(0), p_{1}(0), p_{2}(0)\right)=(.5, .4, .7)$, and all other parameters are the same as in Fig-1. The solution trajectories tend to positive equilibrium $\hat{E}$ and shows that both populations coexist.

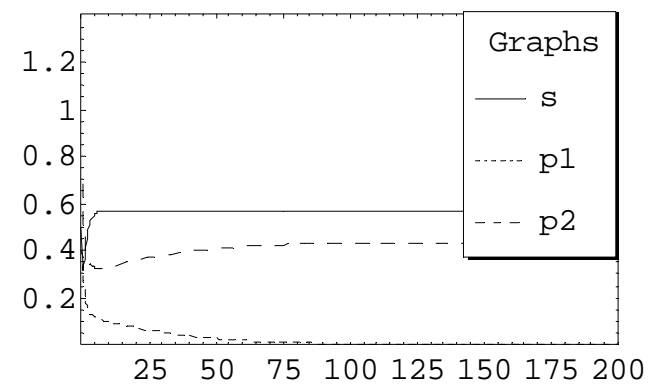

Fig-4: $m_{1}=3.5, b_{1}=.20, c_{1}=.18, m_{2}=2.94$, $b_{2}=.13, c_{2}=.11, D=2.0,\left(s(0), p_{1}(0), p_{2}(0)\right)$ $=(.5, .4, .7)$ The solution trajectories tend to $E_{2}$ and shows that second population avoids extinction.

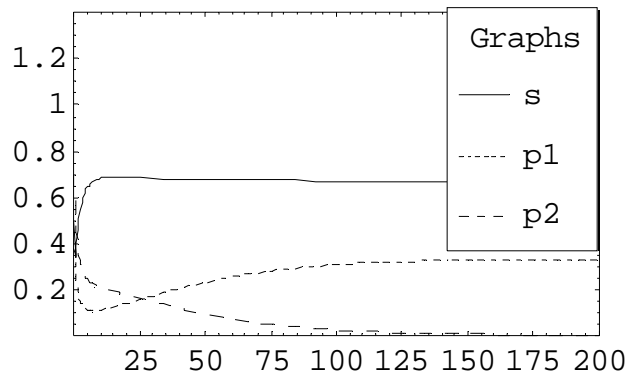

Fig-3: $D=2.2$,

$$
\left(s(0), p_{1}(0), p_{2}(0)\right)=(.5, .4, .7),
$$

and all other parameters are the same as in Fig-1. The solution trajectories tend to $E_{1}$ and shows that first population avoids extinction.

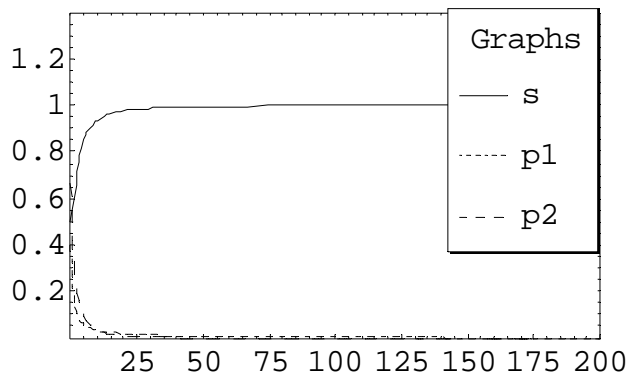

Fig-5: $D=2.5$ and all other parameters are the same as in Fig-4. The solution trajectories tend to $E_{0}$ and shows that both populations extinct.

\section{REFERENCES}

1. R. A. Armstrong and R. McGehee, Competitive exclusion, Amer. Natur., 115 (1980), pp.151-170.

2. K. Gopalsamy, Stability and oscillations in Delay Differential Equations of Population Dynamics, Kluwer Academic Publishers, Dordrecht, 1992.

3. S. B. Hsu, Limiting behavior for competing species, SIAM J. Appl. Math., 34 (1978), pp.760-763.

4. S. B. Hsu, S. Hubbell, and P. Waltman, A mathematical theory of single-nutrient competition in continuous cultures of micro-organisms, SIAM J. Appl. Math., 32 (1977), pp.366-383. 
5. J. N. Kapur, Mathematical Models in Biology and Medicine, New Delhi, 1985, pp.19-41.

6. J. LaSalle, Some extensions of Lyapunov's second method, IRE Trans. Circuit CT-7 (1960), pp. 520 527.

7. G. S. K. Wolkowicz and H. Xia, Global asymptotic behavior of a chemostat model with discrete delays, SIAM J. Appl. Math., 57 (1997), pp. 1019 - 1043. 